\title{
The counterparts of Local Group dwarf spheroidals in nearby clusters
}

\author{
Michael Hilker ${ }^{1}$, Steffen Mieske ${ }^{1}$ \\ and L. Infante ${ }^{2}$ \\ ${ }^{1}$ Sternwarte der Universität Bonn, Auf dem Hügel 71, 53121 Bonn, Germany \\ email: mhilker@astro.uni-bonn.de, smieske@astro.uni-bonn.de \\ ${ }^{2}$ Departamento de Astronomía y Astrofísica, P. Universidad Católica, \\ Casilla 104, Santiago 22, Chile \\ email: linfante@astro.puc.cl
}

\begin{abstract}
In this contribution, we report on the discovery of dwarf spheroidals (dSphs) in the core of the Fornax cluster. Their photometric properties - like magnitude, colour, surface brightness - are very similar to those of Local Group dSphs. However, at a given total magnitude, dSphs in Fornax seem to be more extended than their Local Group counterparts. The membership of several dwarf galaxy candidates in Fornax has been confirmed by surface brightness fluctuation measurements on deep wide-field images taken with the Magellan telescope. The analysis of these images also confirms the flat faint end slope of the luminosity function for dSphs in Fornax which contradicts the expected large number of small dark matter halos connected to dwarf galaxies in $\Lambda$ CDM theory. Dwarf spheroidals have also been detected in the Hydra I and Centaurus cluster. A preliminary analysis of their photometric properties shows that they obey similar scaling relations as their counterparts in Fornax and the Local Group.
\end{abstract}

Keywords. Galaxies: clusters: individual (Fornax, Hydra I, Centaurus), galaxies: dwarf, fundamental parameters, luminosity function, photometry

\section{Introduction}

The identification of dwarf galaxies in different environments plays an important role for the verification of cosmological models. Cold dark matter models predict a large number of small dark matter (DM) halos as sub-structures around Milky Way-sized halos as well as galaxy cluster-sized halos (e.g. Moore et al. 1999). If every small DM halo would contain luminous matter, many dwarf galaxies are expected in basically all environments. However, already in the Local Group there exists a strong discrepancy, the so-called "missing satellite problem" (e.g. Klypin et al. 1999). The faint end of its galaxy luminosity function is quite flat $(\alpha=-1.1$, Pritchet \& van den Bergh 1999) compared with CDM model predictions. Several studies of galaxy clusters show rising numbers of low-luminosity dEs down to about $M_{V} \simeq-11 \mathrm{mag}$ with very different faint-end slopes in the range $-1.1>\alpha>-2.2$ (e.g. Trentham \& Tully 2002). However, it is not known whether the luminosity function in clusters continues to $M_{V}=-8.5$, as it does in the Local Group. Do dSphs actually exist in galaxy clusters? And how abundant are they?

Here, we present the identification and analysis of dwarf spheroidals in nearby clusters, namely the Fornax, Hydra I and Centaurus cluster.

\section{WFCCD survey of dwarf spheroidals in the Fornax cluster core}

End of 1999, we observed the core of the Fornax cluster through the Johnson VI filters with the wide-field CCD (WFCCD) at the 100-inch du Pont telescope at Las Campanas, 

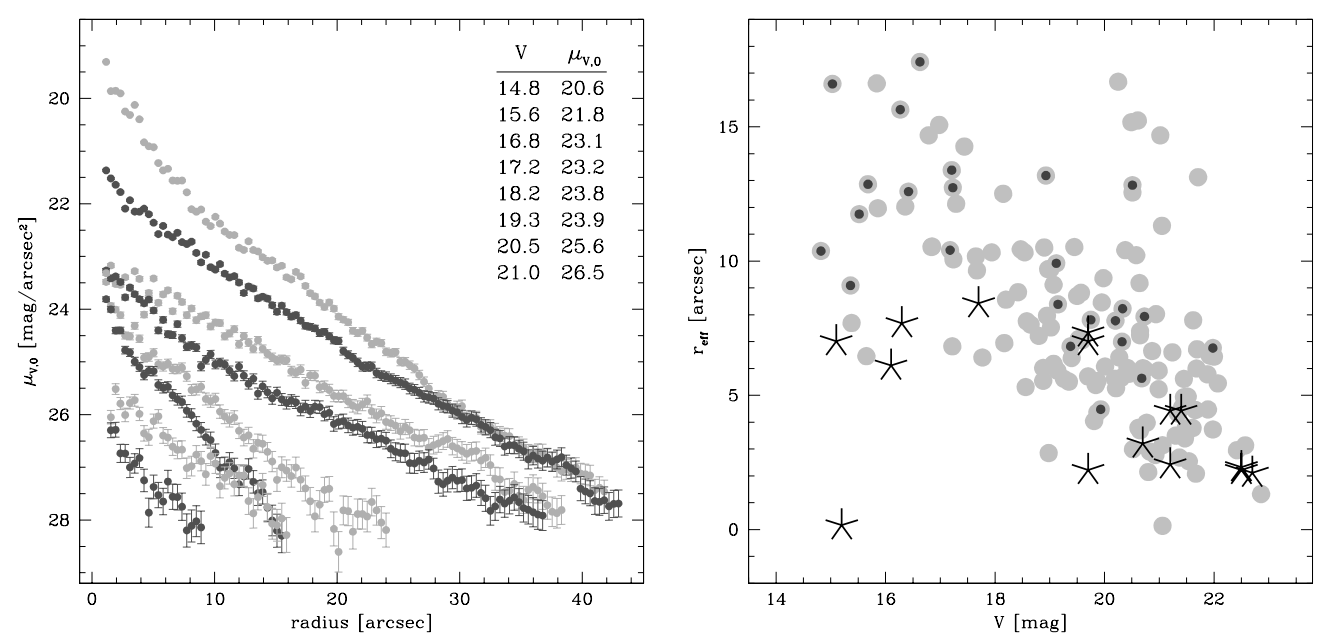

Figure 1. Left: Surface brightness profiles of several dEs and dSphs in the Fornax cluster. The total apparent $V$ magnitudes and central surface brightnesses (exponential law) are indicated. Right: Scale lengths of exponential fits to the outer parts of the surface brightness profiles for dSphs/dEs in Fornax (grey circles) and the Local Group (asterisks) versus $V$ magnitude.

Chile. The pixel scale of 0.77 arcsec/pixel favours the detection of very low surface brightness galaxies. The photometric parameters of the dEs/dSphs have been derived from the analysis of their surface brightness profiles (see Fig. 1): the total magnitude by a curve of growth analysis, the color within an aperture of 8 arcsec diameter, and the central surface brightness from an exponential fit to the outer part of the profile.

First results of our study were presented in Hilker et al. (2003). About 70 new dSph candidates have been discovered beyond the limits of the Fornax Cluster Catalog (FCC, Ferguson 1989), the faintest one with an absolute magnitude of $M_{V} \simeq-8.8$ mag and a central surface brightness of $\mu_{V} \simeq 27 \mathrm{mag} / \operatorname{arcsec}^{2}$. The dSphs follow similar magnitudesurface brightness and also colour-magnitude relations as their counterparts in the Local Group (see Figs. 1 and 2 in Hilker et al. 2003). The faint-end slope of the luminosity function of the dSphs is flat ( $\alpha \simeq-1.1 \pm 0.1$, see Fig. 3 in Hilker et al. 2003).

The only difference between the Local Group dSphs and those in the Fornax cluster are their sizes. Fig. 2 shows that at a given total magnitude, the dEs/dSphs in Fornax are on average larger than their Local Group counterparts (data from Grebel et al. 2003). This is also reflected in the magnitude-surface brightness diagram (Fig. 3) where the Local Group dwarfs have on average a higher central surface brightness than the Fornax dSphs. This finding might be explained by environmental effects, i.e. the strong tidal forces in a cluster that lead to more extended, lower surface brightness dwarf galaxies, and finally to a destruction of dSphs close to the cluster center (e.g. Hilker et al. 1999).

\section{SBF measurements in the Fornax Deep Field}

The WFCCD study has shown that the combination of deep multi-color photometry in a wide field with a sufficient resolution is crucial in order to identify dwarf spheroidal candidates in nearby clusters. However, one still can not be sure that all dSph candiates are real cluster members, as long as one has not measured their distances.

Therefore, to improve the membership assignment, we have re-imaged the central Fornax cluster with substantially better spatial resolution ( $0.2 \mathrm{arcsec} / \mathrm{pixel})$ using IMACS and Magellan at LCO. From the first part of our survey, we directly determined the 

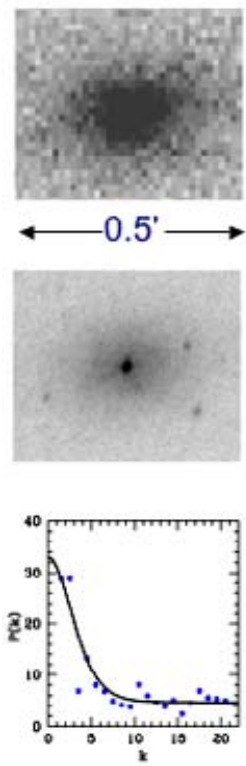
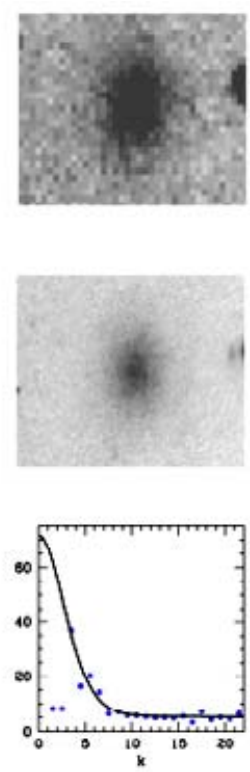
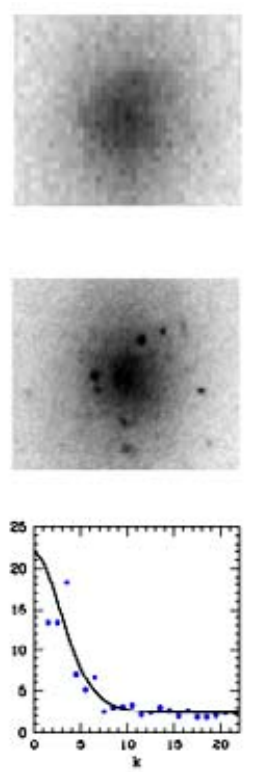
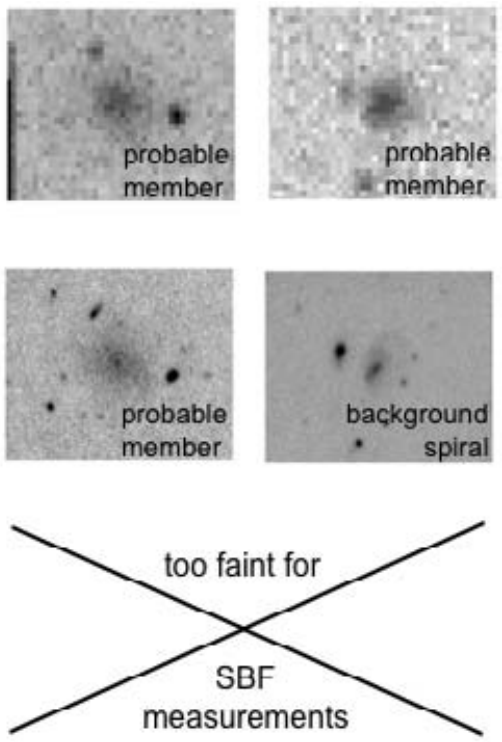

Figure 2. Left: three examples for SBF measurements in dEs. The top and middle row show thumbnail images galaxies from the WFCCD and IMACS data sets, respectively. In the bottom row the azimuthally averaged power spectra of the SBF measurement and the corresponding fits are plotted. Right: four galaxies are shown that are too faint or small for SBF measurements.

cluster membership for 10 previously unconfirmed candidate dEs in the magnitude range $-14.2<M_{V}<-11$ mag using the SBF method (Fig. 2, see Mieske et al. 2003 for details of this method). This extends the magnitude range of confirmed cluster members far into the regime where the faint end slope $\alpha$ dominates the galaxy luminosity function.

Furthermore, we improved the morphological cluster membership assignments for fainter galaxies $\left(M_{V}<-10 \mathrm{mag}\right)$. For the vast majority of $\mathrm{dE}$ candidates we confirm the probable cluster membership, such that $\alpha$ changes by less than 0.02 compared to the previously determined value. Only very few dSph candidates turn out te be likely background galaxies due to their better resolved morphology (see Fig. 2). We find two new dSph candidates from our IMACS imaging (see Fig. 3). Including them does not change $\alpha$, either. This further confirms the strong discrepancy between the number of low mass dark matter halos expected in a $\Lambda$ CDM universe and the number of low luminosity galaxies.

The new membership determinations from the first part of the "Fornax Deep Field" survey are summarised in the magnitude-surface brightness plot (see Fig. 3). Except for a few barely resolved galaxies (close to the dashed line), the morphological membership classification seems to work very reliable.

\section{Dwarf spheroidals in the Hydra I and Centaurus cluster}

The central parts of the HydraI and Centaurus cluster have been imaged through Johnson $V I$ with FORS1 at the VLT in dark time and under excellent seeing conditions (for first results, see Mieske \& Hilker 2003). A first visual inspection of the images revealed a wealth of faint, spatially resolved dSph candidates. The surface brightness profiles of a small random sample has been measured. Their structural and photometric parameters are such that they fall on top of the colour-magnitude and magnitude-surface brightness relation defined by the Fornax dSphs. The publication of these results is in preparation. 


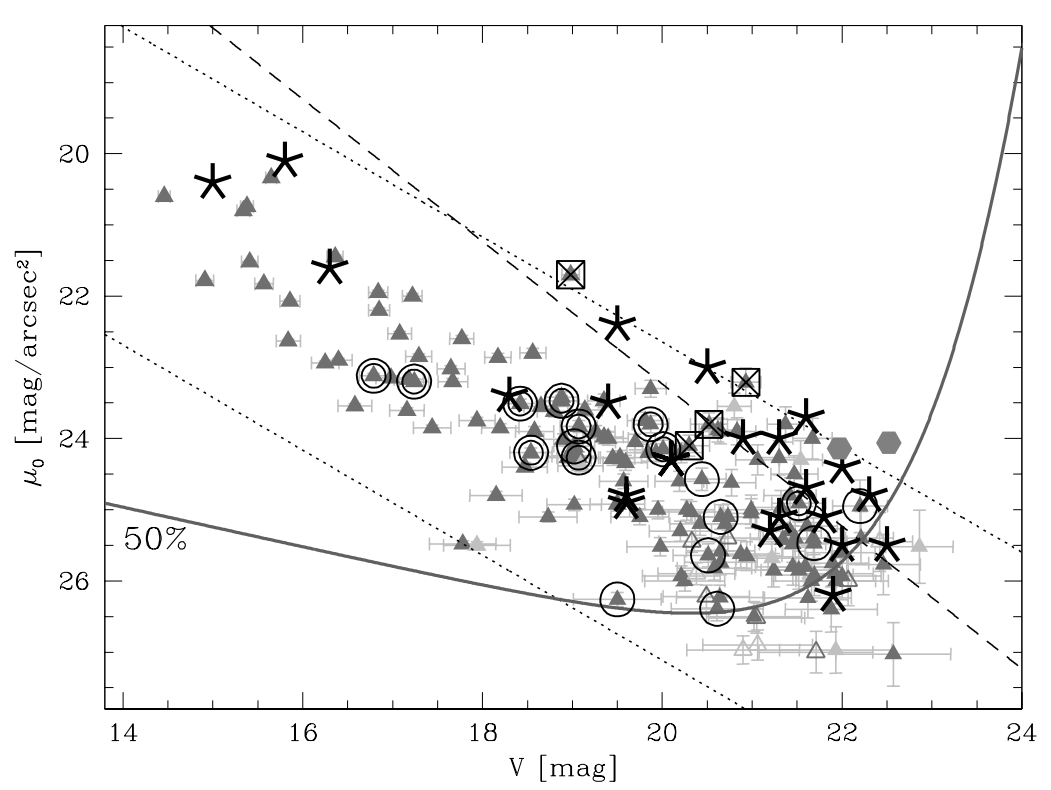

Figure 3. Magnitude-surface brightness diagram of dEs and dSphs in the Fornax cluster (triangles) and the Local Group (asterisks). Light grey triangles mark galaxies that lie outside $3 \sigma$ of the colour-magnitude relation, open triangles those galaxies that were identified only by eye. Double circles indicate cluster membership confirmed with SBF, single circles probable membership due to morphology. Probable background galaxies (due to morphology) are crossed out. The two filled hexagons mark two additional cluster member candidates found from the IMACS imaging. The dashed line indicates a scale length of 2.5 arcsec for an exponential profile. The dotted lines are the $3 \sigma$ deviations from the magnitude-surface brightness relation as defined by the Fornax dEs. The solid curve shows the completeness limit of $50 \%$.

\section{Conclusions}

1. The counterparts of Local Group dSphs do exist in Fornax. They follow similar colourmagnitude and magnitude-surface brightness relations, except that the Fornax dSphs seem to be in average larger at a given total magnitude than the Local Group dSphs.

2. SBF-cluster memberships and improved morphological classifications do not change the shallow faint end slope of $\alpha \simeq-1.1$ derived from the WFCCD-data by more than 0.02 , posing a challenge to $\Lambda \mathrm{CDM}$ theory.

3. Preliminary analysis of deep VLT images in Hydra I and Centaurus show that dSph candidates exist in these clusters, and that they follow similar scaling relations as their counterparts in Fornax. More on this in forthcoming publications.

\section{References}

Ferguson, H.C. 1989, AJ 98, 367

Grebel, E.K., Gallagher, J.S. \& Harbeck, D. 2003, AJ 125, 1926

Hilker, M., Infante, L. \& Richtler, T. 1999, A\&AS 138, 55

Hilker M., Mieske S. \& Infante L. 2003, A\&A (Letters) 397, L9

Klypin, A., Kravtsov, A.V., Valenzuela, O. \& Prada, F. 1999, ApJ 522, 82

Mieske, S. \& Hilker, M. 2003, A $\mathscr{E} A$ 410, 445

Mieske, S., Hilker, M. \& Infante, L. 2003, A\& $A$ 403, 43

Moore, B., Gighna, S., Governato, F., et al. 1999, ApJ (Letters) 524, L19

Pritchet, C.J. \& van den Bergh, S. 1999, AJ 118, 883

Trentham, N. \& Tully, R.B. 2002, MNRAS 335, 712 


\section{Discussion}

Moore: The luminosity function you find is similar to the Local Group, suggesting a comparable "missing satellite problem". In a scenario where reionisation cuts off small galaxy formation you would expect more dwarf galaxies - comments?

HILKER: Right, if there originally existed a large number of small galaxies in Fornax, the nowadays flat luminosity function of $\mathrm{dEs} / \mathrm{dSphs}$ requires evolutionary processes that reduced the number of small galaxies in the cluster center, like for example tidal disruption. The decline in number counts of dEs in the very center of the cluster might be a hint for this. However, maybe the reionisation scenario can be adjusted in such a way that no/less small galaxies have formed (and the nowadays existing dSphs have a different origin?).

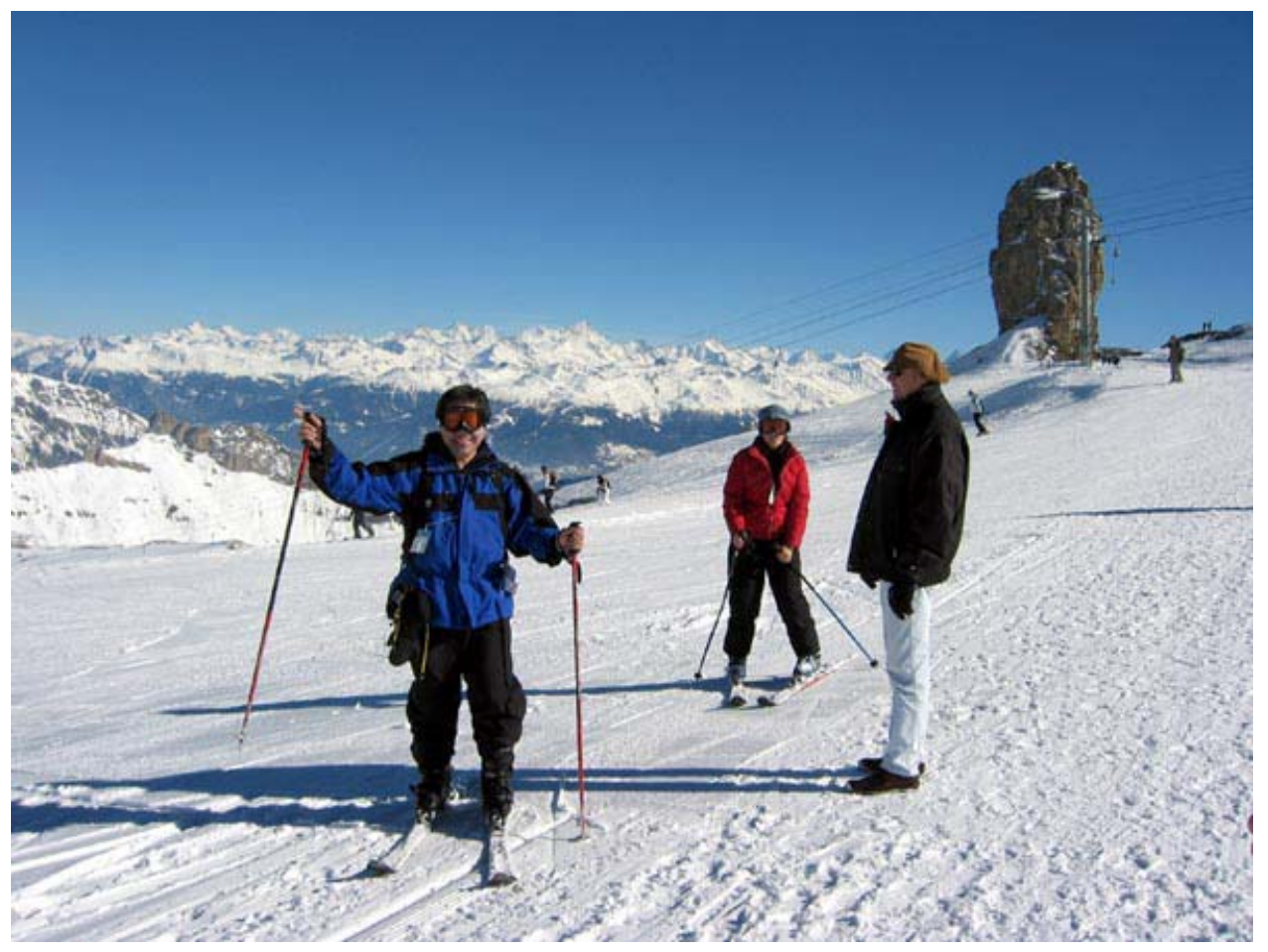

